\title{
Phytonutrient intake and body composition: Considering colors
}

\author{
Ryan Orgeron II*, Janet Pope, Vicky Green, Dawn Erickson
}

School of Human Ecology, Louisiana Tech University, Ruston, LA, USA

Submission date: November $12^{\text {th }}, 2018$, Acceptance Date: February $25^{\text {th }}$, 2019, Publication Date: February $28^{\text {th }}, 2019$

Citation: Orgeron R, Pope J, Green V, Erickson D. Phytonutrient intake and body composition. Functional Foods in Health and Disease 2019; 9(2): 108-122. DOI: https://doi.org/10.31989/ffhd.v9i2.583

\begin{abstract}
Background: Being overweight or obese is one of the most harmful risk factors to the health of a population. The cause of obesity is complex and multifactorial, but a primary cause is a nutrientpoor diet. Despite excess intake of calories, diets of obese individuals commonly lack phytonutrients. Phytonutrients such as chlorophyll, carotenoids, and anthocyanins have all been shown to have anti-obesity effects. National data show that populations with the lowest intakes of fruit and vegetable intake also have higher obesity rates. Available data suggests that a low fruit and vegetable intake, corresponding to a low phytonutrient intake, is correlated with excess adiposity.
\end{abstract}

Objective: To determine if phytonutrient intake is correlated with body composition and to examine correlations between certain colors of fruits and vegetables.

Methods: Fifty individuals from Thibodaux, Louisiana were randomly selected to participate in this study. Three 24-hour food recalls were used to calculate phytonutrient content of the diets using the phytonutrient index (PI). A food frequency questionnaire was used to determine intake of specific phytonutrients (carotenoids, anthocyanins, and carotenoids). Body adiposity was measured using body mass index (BMI), waist circumference, and fat percentage. Pearson correlation was used to assess correlations between phytonutrient index and body adiposity measures. Analysis of variance (ANOVA) with post-hoc tests was used to determine differences in PI and phytonutrient intakes among the BMI, waist circumference, and percent body fat groups.

Results: Participants who met healthy standards for BMI, waist circumference, and fat percentage all averaged significantly higher PI scores compared to those classified as obese/overweight. A strong inverse relationship was found between PI and BMI $(r=-0.753, p=0.00)$, waist circumference $(r=-0.730, p=0.00)$, and body fat percentage $(r=-0.701, p=0.00)$. Individuals with higher weekly intakes of chlorophyll, carotenoid, and anthocyanin-rich foods had better body composition compared to those who consumed less $(\mathrm{p}<0.05)$. 
Conclusion: Results suggest a strong correlation between higher phytonutrient intake and improved body composition. Overall diet quality seems to make the most difference, but phytonutrients are likely a key reason for those benefits.

\section{BACKGROUND}

Obesity is believed to be a root cause of many prominent diseases [1, 2, 3]. While the cause of obesity is complex and multifactorial, a likely factor is poor nutrition [4, 5]. Research has shown that despite excess intake of calorie-dense foods, a common dietary deficiency among individuals with excess adiposity is the lack of phytonutrients [6]. Thus, adequate intake of phytonutrients could also be a significant factor in improving excess body adiposity [6, 7].

The Centers for Disease Control and Prevention (CDC) data indicate over one-third of the United States population is obese [8]. Data from the Behavioral Risk Factor Surveillance System (BRFSS) show that nearly 70\% of Louisiana adults are overweight or obese [9].

Data from the CDC indicates that $46.7 \%$ of Louisiana's population eats less than one serving of fruit and $32.5 \%$ eat less than one serving of vegetables each day [10]. Many Louisianans do not consume one serving per day, much less the five servings per day recommended by the United States Department of Agriculture [11]. These data suggest that a low fruit and vegetable intake, thus low phytonutrient intake, is correlated with excess adiposity $[8,9,10]$.

Research has shown a strong correlation between low fruit and vegetable (F/V) intake and excess adipose tissue [4]. A low intake of these foods is associated with higher energy intake and reduced nutrient intake $[2,5]$. Thus, a lack of $\mathrm{F} / \mathrm{V}$ consumption and/or phytonutrients (plant nutrients) could potentially be a major contributor to Louisiana's high obesity rates.

Despite researchers isolating phytonutrients in studies, research shows that these nutrients elicit benefits best when left in whole foods $[12,13]$. Moreover, instead of isolating phytonutrients, studying them in their whole form based on color could be a more efficient method [13]. This is due to phytonutrients displaying specific pigments when abundant in the plant [14].

Phytonutrients of particular interest are chlorophyll, carotenoids, and anthocyanins. Current research shows potential for chlorophyll as a therapeutic phytonutrient that can help treat and/or prevent adiposity $[15,16,17]$. Anthocyanins may play a role in combating obesity by stimulating reductions in adipose tissue through various pathways $[18,19,20]$. Carotenoids have been found in a variety of studies to play a role in the prevention of excess adiposity. Although human trials are scarce, they have indicated a beneficial effect of increased carotenoid intake on adiposity [21, 22].

Very few clinical intervention trials have been properly designed and executed due to these studies being difficult to conduct [23]. The data from observational studies are more likely to suggest a role for phytonutrients in the prevention and treatment of obesity [18]. In the future, new and sufficient models that would allow the design to be more consistent and more efficient for clinical studies are needed [23, 24].

In an effort to study phytonutrients in an easily accessible and inexpensive way, a method called the "Phytochemical Index" (PI) was proposed [25]. Although it is not the most precise method available, it can provide a valid estimation of phytonutrient intake, which can aid in studying the health correlations of diets high and/or low in phytonutrient-rich foods [25, 26, 27, 28]. 
There are many methods in which adiposity and obesity can be measured. The most commonly used method is the BMI $[29,30]$. Another common anthropometric measure of adiposity is waist circumference, which is often shown to be more efficient than BMI alone. Interestingly, using BMI and waist circumference in tandem has been shown to offset limitations of each [31, 32, 33]. Despite being simple and effective methods, BMI and waist circumference are only predictors of adiposity and do not actually measure body composition. Bioelectrical impedance analysis (BIA) is more accurate than body mass index and most other anthropometric measures in assessing body composition [34]. When compared to more advanced measures, BIA is less expensive, less invasive, and easier to use [34, 35].

\section{METHODS}

This cross-sectional study investigated correlations between PI correlations and BMI, waist circumference and percent body fat. A second aim was to examine if correlations existed between certain colors of fruits and vegetables (green, red/purple, and yellow/orange) and fat percentage, waist circumference, and BMI. Institutional Review Board approval was obtained from the Thibodaux Regional Wellness Center and the Louisiana Tech University Human Use Committee.

\section{Participants}

Fifty (50) participants were randomly selected from Thibodaux Regional Wellness Center in Thibodaux, Louisiana, through computerized simple random sampling. Inclusion criteria required the participants to be between the ages of 18 and 55. Due to uncertain validity of body fat measures in morbidly obese subjects $\left(>40 \mathrm{~kg} / \mathrm{m}^{2}\right)$, subjects with a BMI $>40 \mathrm{~kg} / \mathrm{m}^{2}$ were excluded [36]. Due to the use of electrical currents in the bioelectrical impedance analysis (BIA), pregnant subjects were also excluded. Participants were required to give informed consent prior to being enrolled in the study.

\section{Body Adiposity Assessments}

The instrument used to assess body adiposity was a Tanita ${ }^{\mathrm{TM}}$ BF-350 bioelectrical impedance analysis (BIA) scale. The scale was linked to a desktop computer with the program Polar Body $\mathrm{Age}^{\mathrm{TM}}$ in order to assess body composition. The linked Tanita ${ }^{\mathrm{TM}}$ BF-350 and the Polar Body $\mathrm{Age}^{\mathrm{TM}}$ program calculated BMI and body fat percentage. Waist circumference was measured with a flexible tape, and a mounted wall measuring tape was used to measure height. The BIA scale accurately weighs up to 440 pounds. However, body fat percentage could be overestimated in patients who are dehydrated due to BIA scales assuming normal hydration [36]. Participants were given a protocol sheet when scheduled for assessment to promote proper hydration the day before and the day of their assessment.

\section{Nutrition Assessment}

All subjects participated in three-pass 24-hour food recalls, which were used to estimate the phytonutrient index score. Recalls were obtained from two weekdays and one weekend day to acquire an estimate of their daily intake [28]. All food recalls were administered face-to-face and one-on-one with each participant by the primary investigator. Each food recall was done within 24 hours of the day being recalled. In order to estimate serving sizes of foods consumed, standard measuring tools were used to give visual amounts to better predict intake. The primary investigator 
(a registered dietitian), probed for details in individual consumption to get as accurate estimations as possible. For example, if a participant recalled eating a sandwich, the dietitian researcher asked follow-up questions to recall which smaller components made up the sandwich such as tomatoes, mustard, lettuce, etc.

In order to determine different sources of phytochemicals by color, each participant was also administered a Food Frequency Questionnaire (FFQ) to determine the intake of the specific color classifications (green, purple, and orange). Example foods were presented to participants to allow a more accurate measure of frequency. Although there is no gold standard to FFQ categories, the categories used by the National Health and Nutrition Examination Survey were reflected [37, 38]. The categories include less than once per month, 1-3 per month, once per week, 1-3 per week, 46 per week, once per day, and 2-3 per day. These servings were averaged for data analysis so that all were reported as weekly intakes without ranges. Less than once per month and 1-3 per month were recorded as 0.5 servings/week; 1-3/week was reported as 2/week; 4-6/week were reported as 5/week, 1/day was reported as 7/week; and 2-3/day was reported as 17.5/week. In order to control for seasonal intake (i.e. fruits and vegetables), the FFQ represented intake on an annual basis and that was explained to each participant.

\section{Procedures}

Subjects were screened in the member computer system in order to identify those meeting age and BMI inclusion criteria. Subject selection was done through computerized simple random sampling. After selecting subjects, their health questionnaire and doctor notes were pulled to confirm that they were not on obesity-related medications. Once eligibility was determined for the study, individuals were contacted for verbal and written informed consent and participation acceptance. Once participation was accepted, subjects were scheduled to come into the clinic for their body composition and nutrition assessment.

The first part of the assessment was measuring height for BMI. Afterwards, waist circumference was measured following the World Health Organization (WHO) approved protocol [39]. The Tanita ${ }^{\mathrm{TM}}$ BF-350 BIA scale was used to provide weight (in pounds), BMI, and body fat composition (fat mass and lean mass).

The nutrition assessment consisted of three 24-hour recalls by a Registered Dietitian in a oneon-one setting with each subject. The dietitian recorded all of the recalled data (meals, beverages, snacks, etc.). To increase accuracy of food quantity, household measures were used as visuals during the food recall. Each recall was done on separate days and within 24 hours of the day being recalled.

The total energy intake (estimated with Elizabeth Stewart Hands and Associates (ESHA) software) was used for the PI calculation. The PI estimated how much of the subject's energy intake was accounted for by phytonutrient rich foods. The formula for the PI is [(phytochemicalrich foods $\mathrm{kcal} /$ total daily energy intake $\mathrm{kcal}) \times 100$ ]. Phytonutrient rich foods primarily include fruits and vegetables, but also legumes, whole grains, seeds, nuts, olive oil, soy sources, and wine [25]. In addition to calculating the PI, the dietitian also identified intakes of the specific colors (green, orange, and purple) through a FFQ. More specifically green colored plants are chlorophyllrich (such as spinach, broccoli, celery, etc.), reddish-purple foods are anthocyanin-rich (such as purple cabbage, grapes, blueberries, etc.), and orange-yellowish foods are carotenoid rich (such as carrots, sweet potatoes, mangoes, etc.) $[13,14]$. 


\section{Statistical analysis}

IBM SPSS Statistics version 24 was used to analyze the data. Background data including age, sex, health status, and race were summarized, and the Pearson correlation was used to assess correlations between phytonutrient index scores and body adiposity measures. Analysis of variance (ANOVA) with post-hoc tests was used to determine differences in PI and phytochemical intakes among the BMI, waist circumference and percent body fat groups. Results were considered statistically significant if p-value was less than or equal to 0.05 .

\section{RESULTS}

Fifty (50) participants were recruited to participate in the study and none of them declined. All 50 participants remained until the completion of the study. Table 1 provides a description of the subjects in the study including sex, ethnicity, and current health diagnosis. The average age was $36.8 \pm 11.4$ years with a range of 18 to 54 years. Despite having a variety of ethnicities, almost $75 \%$ of the population identified as White/Caucasian and $70 \%$ of the population was female. Nearly half of the sample had some form of health condition (most notably diabetes and hypertension). Demographic characteristics of the group are summarized in Table 1.

Table 1. Demographics of Participants $(n=50)$

\begin{tabular}{lcc}
\hline Demographic & $(n)$ & $\%$ \\
\hline Gender & 15 & $30 \%$ \\
Male & 35 & $70 \%$ \\
Female & & \\
& & \\
Ethnicity & 37 & $74 \%$ \\
White/Caucasian & 9 & $18 \%$ \\
African American & 4 & $8 \%$ \\
Latino & & \\
& & \\
Health Diagnosis & 8 & $16 \%$ \\
Hypertension & 9 & $6 \%$ \\
Type 2 Diabetes & 3 & $6 \%$ \\
Hyperlipidemia & 3 & $2 \%$ \\
Osteoarthritis & 1 & \\
Cystic Fibrosis & & \\
\hline
\end{tabular}

The mean PI score and average caloric intake of the entire group was $38.1 \pm 21.1$ and 1,271 \pm 393 , respectively. Participants with various health conditions had average phytonutrient index (PI) 
lower than the population average This was true of participants with type 2 diabetes $(22 \pm 17.7)$, hypertension $(17.4 \pm 11.6)$, hyperlipidemia $(21.6 \pm 19.4)$, and osteoarthritis $(24.3 \pm 27.4)$. Waist circumference and body fat percentage categorized nearly half of the participants as having healthy body composition (waist circumference $=27 / 50$; body fat $=24 / 50$ ), while the body mass index (BMI) categorized 17 out of 50 as healthy. Table 2 summarizes the average phytonutrient index (PI) of the participants in each category of body composition measures as well as significant differences between categories based on Analysis of Variance (ANOVA).

Table 2 Average Phytonutrient Index of Participants $(n=50)^{1}$

Body Composition Measures

Body Mass Index $\left(\mathrm{kg} / \mathrm{m}^{\mathbf{2}}\right)$

Healthy

Overweight

$(18.5-24.9)$

Obese

Severely Obese $\quad(35-39.9)$

\section{Waist Circum ference ${ }^{2}$}

Healthy - meets standard

Overweight - over standard

\section{Body Fat Percentage ${ }^{3}$}

Healthy

Overweight

Obese

(n) $\quad$ Mean \pm SD

17

9

12

11

27

23

24

11

15
$53 \pm 12.4^{\text {a }}$

$57 \pm 10.8^{\text {a }}$

$20 \pm 14.4^{b}$

$18 \pm 7.7$ b

Phytonutrient index represents the percentage of diet that comes from phytonutrient-rich foods ${ }^{2}$ Meets standard in waist circumference is 40 inches or less for males and 35 inches or less for females.

${ }^{3}$ World Health Organization guidelines for body fat percentage are based on gender and age of individual. $\stackrel{a b, c}{ }$ different superscripts indicate values are significantly different $(\mathrm{p}<.05)$

Statistically significant correlations were found between the PI ratings and body composition ranges. The only measure that did not follow the trend for positive correlations between PI and lower body adiposity was body mass index. Participants with an overweight BMI had a higher PI average than those with a healthy BMI. Overall, the data analysis showed a strong inversecorrelation between PI and BMI $(r=-0.753, p=0.00)$, waist circumference $(r=-0.730, p$ $=0.00)$, and body fat percentage $(\mathrm{r}=-0.701, \mathrm{p}=0.00)$. Figures 1,2 , and 3 show the correlation graphs. 


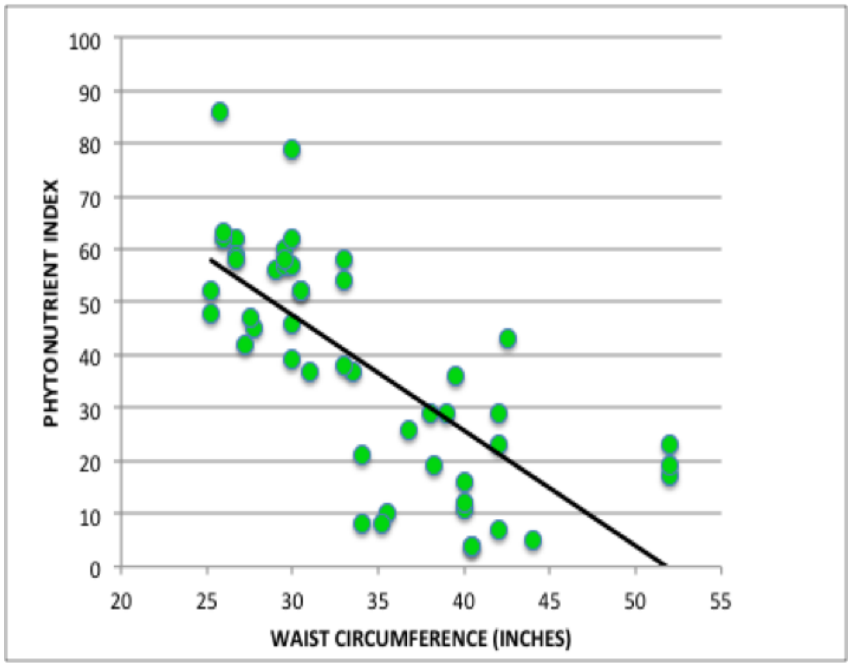

Figure 1: Phytonutrient Intake and Waist Circumference

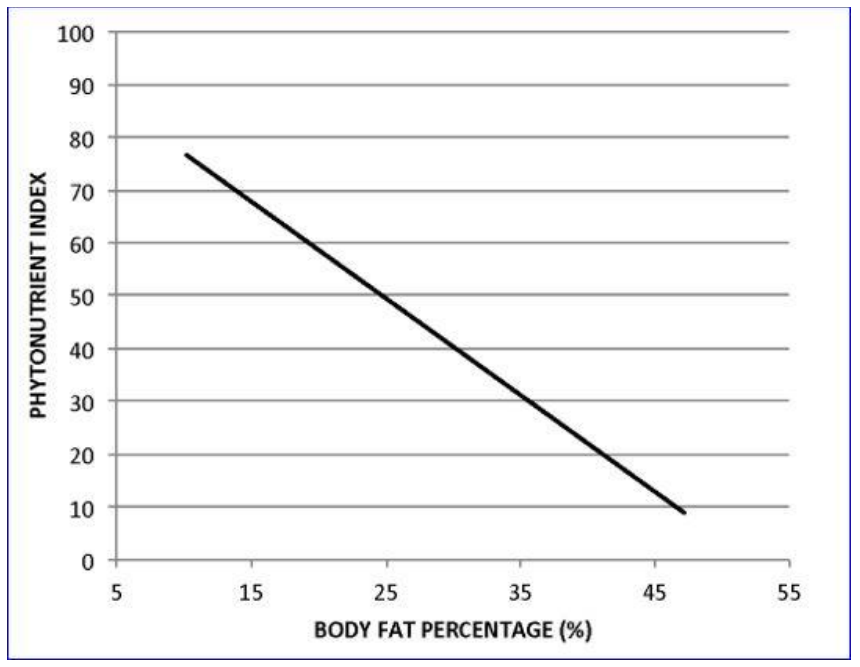

Figure 2: Phytonutrient Intake and Body Fat Percentage

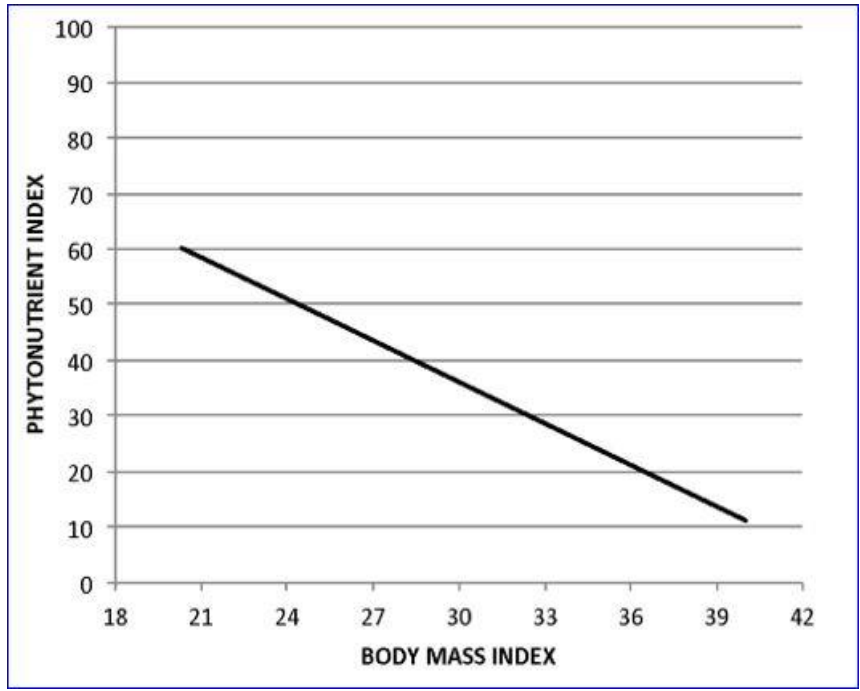

Figure 3: Phytonutrient Intake and Body Mass Index

ANOVA testing was used to analyze significant differences $(p<.05)$ in the intake of foods containing specific types of phytonutrients (chlorophyll, carotenoids and anthocyanin) based on 
body composition measures (shown in Tables 3, 4, and 5). All the standards of body composition showed significant differences $(\mathrm{p}<.05)$ except for the "healthy" and "overweight" standards measured by BMI.

Individuals with "healthy" body fat percentages had significantly greater intakes of all phytonutrient types than those with excess body fat (overweight and obese). However, there was not a significant difference in intakes between "overweight" and "obese" body fat percentages. The waist circumference standards followed the same trend as those with "healthy" waist sizes had significantly greater intakes of all phytonutrients in comparison to those that exceeded healthy waist sizes.

Considering BMI measurements, individuals that were within "healthy" standards had significantly greater intakes of all phytonutrient types when compared to those within "obese" and "severely obese" standards. However, there was no significant difference between the intakes of those with "healthy" and "overweight" standards.

\begin{tabular}{|c|c|c|}
\hline \multicolumn{2}{|c|}{ Body Composition } & Mean \pm SD \\
\hline \multicolumn{3}{|c|}{ Body Mass Index $\left(\mathrm{kg} / \mathrm{m}^{2}\right)$} \\
\hline Healthy & $(18.5-24.9)$ & $7.0 \pm 5.4^{\mathrm{a}}$ \\
\hline Overweight & $(25-29.9)$ & $8.9 \pm 6.1^{\text {a }}$ \\
\hline Obese & $(30-34.9)$ & $2.5 \pm 2.5^{b}$ \\
\hline Severely Obese & $(35-39.9)$ & $1.8 \pm 1.8^{b}$ \\
\hline \multicolumn{3}{|c|}{ Waist Circum ference } \\
\hline \multicolumn{2}{|c|}{ Healthy - meets standard } & $7.5 \pm 5.7^{\text {a }}$ \\
\hline \multicolumn{2}{|c|}{ Overweight - over standard } & $2.3 \pm 2.3 b$ \\
\hline \multicolumn{3}{|c|}{ Body Fat Percentage } \\
\hline \multicolumn{2}{|c|}{ Healthy } & $8.5 \pm 5.5^{\text {a }}$ \\
\hline \multicolumn{2}{|l|}{ Overweight } & $2.6 \pm 2.0 \mathrm{~b}$ \\
\hline \multicolumn{2}{|l|}{ Obese } & $1 . \overline{7 \pm} 1.8 \mathrm{~b}$ \\
\hline \multicolumn{3}{|c|}{$\begin{array}{l}1 \text { The intake means are based off of how many servings per week were consumed } \\
\text { c different superscripts indicate values are significantly different }(\mathrm{p}<.05)\end{array}$} \\
\hline
\end{tabular}




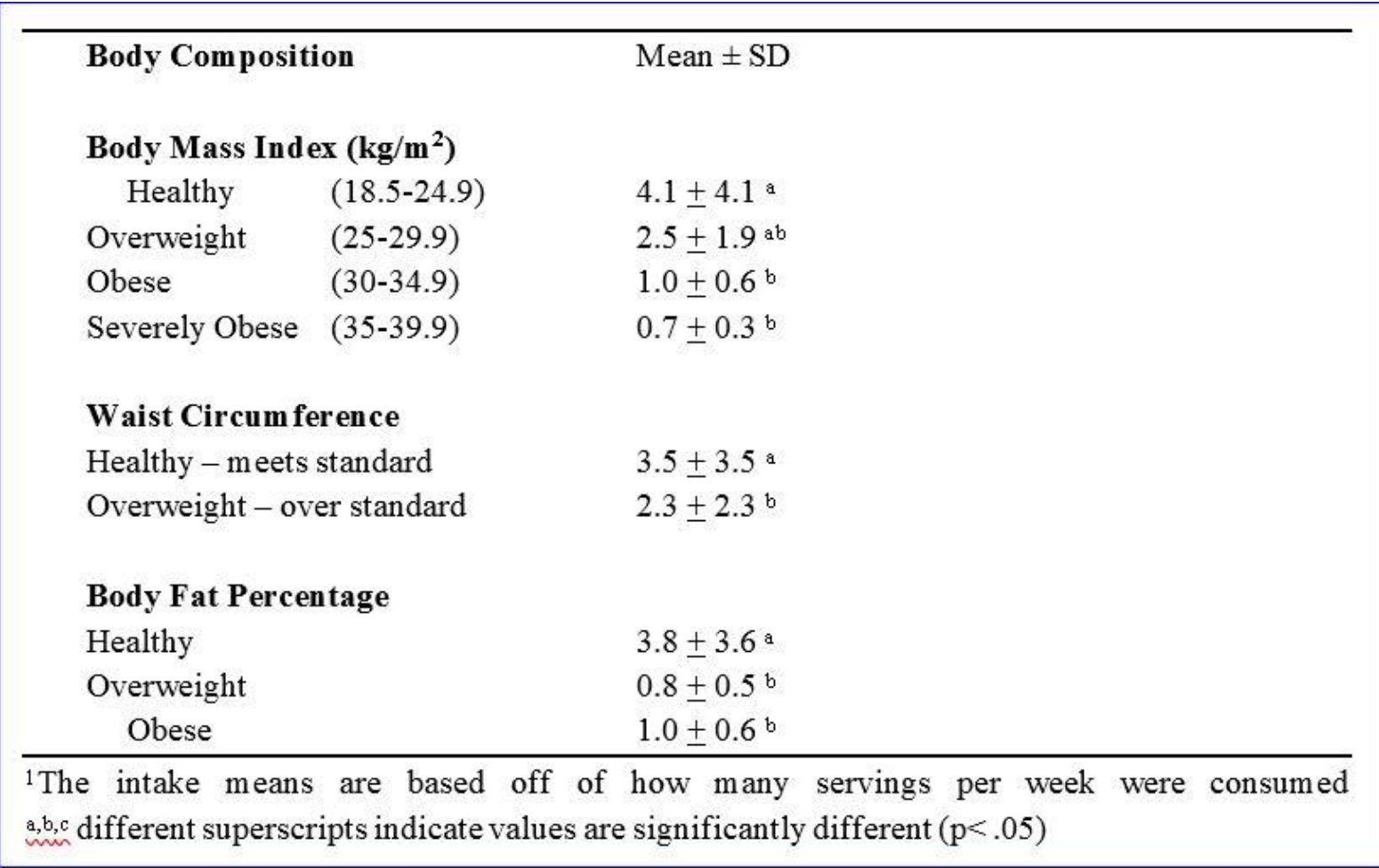

Table 5 Mean Daily Servings of Anthocyanin Foods Based on BMI, Waist Circumference, and Percent Body Fat

\section{Body Composition}

Body Mass Index $\left(\mathrm{kg} / \mathrm{m}^{2}\right)$

Healthy

Overweight

Obese

$(18.5-24.9)$

$(25-29.9)$

Severely Obese $\quad(35-39.9)$

\section{Waist Circum ference}

Healthy - meets standard

Overweight - over standard

\section{Body Fat Percentage}

Healthy

Overweight

Obese
Mean \pm SD

$$
\begin{aligned}
& 4.0 \pm 2.9^{a} \\
& 3.5 \pm 2.5 a b \\
& 0.9 \pm 0.5^{b c} \\
& 0.7 \pm 0.5^{c}
\end{aligned}
$$

$$
3.7 \pm 2.8^{\text {a }}
$$$$
1.0 \pm 0.6^{b}
$$
$4.1 \pm 2.7^{\text {a }}$
$1.1 \pm 0.7$ b
$0.7 \pm 0.4^{b}$

1 The intake means are based off of how many servings per week were consumed a,b,c different superscripts indicate values are significantly different $(\mathrm{p}<\quad .05)$ 


\section{CONCLUSION}

An abundance of nutrition and scientific research leads to the conclusion that higher intakes of F/V are correlated with improvements in anthropometric parameters and preventing/treating adiposity $[4,40,41]$. However, some research has indicated there is more to fruits and vegetables than fiber and vitamins, and that phytonutrients could also be a primary component of food contributing to the improvement in body composition [6,24]. The purpose of this study was to determine if phytonutrient intake measured by the PI is correlated with body composition. Data were collected on 50 individuals from Thibodaux Regional Wellness Center in Thibodaux, Louisiana. The data showed that higher intakes of phytonutrients significantly correlate with better body composition in all three measures (body mass index, waist circumference, and body fat percentage). BMI categorized the least amount of participants as healthy (17/50). It is a possibility that individuals with more muscle/bone density had BMIs classifying them in the overweight category despite having low body-fat and waist circumference. The population in Louisiana is particularly interesting because nearly $70 \%$ of Louisiana adults are overweight or obese and reportedly consume low amounts of fruits and vegetables [9, 10]. According to the NHANES, American males consume an average of 2,640 calories/day and females consume an average of 1,785 calories/day (average of 2213 for both genders). The data on this population sample averaged a much lower intake of daily calories at $1271 \pm 393$. Many of the participants (21/50) reported eating only once or twice per day, consuming as little as 565 calories per day. This data further suggests that calorie consumption is not the only factor influencing obesity. However, the United States Department of Agriculture states that many respondents under report intake and that studies show actual intakes may be considerably higher.

Every participant with a health condition had a lower PI score than the population average $(38.1 \pm 21.1)$ except for one who had cystic fibrosis $(\mathrm{PI}=57)$. This exception is likely related to cystic fibrosis being a genetic condition not developed by lifestyle factors (e.g., diet). This data supports previous studies showing that increased fruit and vegetable intake has been correlated with reduced risk of diseases [42, 43, 44]. Although a small sample, these data are some of the first to show any relationship between PI and disease prevalence.

In an effort to determine differences in the intakes of specific phytonutrients (chlorophyll, carotenoids, and anthocyanin) based on body composition, one-way ANOVA testing was used. Phytonutrient intake was not precisely measured, but rather identified by categorizing foods by color as some research supports due to evidence that whole food is more applicable and health promoting than precisely isolated phytonutrients $[12,13]$.

Individuals with "healthy" body fat percentages had significantly greater intakes of all three phytonutrient types than those with excess body fat (overweight and obese). However, there was not a significant difference in intakes between "overweight" and "obese" body fat percentages. The waist circumference standards followed the same trend as those with "healthy" waist sizes had significantly greater intakes of all phytonutrients in comparison to those that exceeded healthy waist sizes.

Considering BMI measurements, individuals that were within "healthy" standards had significantly greater intakes of all phytonutrient types when compared to those within "obese" and 
"severely obese" standards. However, there was no significant difference between the intakes of those with "healthy" and "overweight" standards.

Interestingly, each body composition measure had a different phytonutrient that showed the most significant difference. For BMI measurements, the intake of carotenoids between "healthy" and "severely obese" individuals showed the most significance $(\mathrm{p}<0.00)$. The intake of anthocyanin showed the most significant difference between standard and excess waist sizes ( $\mathrm{p}<$ $0.00)$. Chlorophyll intakes between those with "healthy" and "obese" body fat percentages showed most significant differences $(\mathrm{p}<0.00)$. Further analysis showed that participants who had higher intakes of one phytonutrient typically had higher intakes of others which skews any evidence that one has any more of an effect than others.

The cause of obesity is very complex, but poor nutrition seems to be a primary cause $[4,5]$. Fruit and vegetable intake has long been shown to have a positive effect on health and body composition [41, 42, 45, 46]. Researchers are not clear as to why fruits and vegetables have this effect, but some studies have shown varying body composition changes among subjects depending on which fruits and vegetables they consumed more often [47, 48]. In considering the differences between various plant foods, a major difference is the specific phytonutrients they contain.

Research has shown that despite excess intake of calorie dense foods, obese individuals lack phytonutrient intake $[6,7]$. These phytonutrients are primarily sourced from fruit and vegetable intake $[6,7]$.

In a population like Louisiana, data shows that less than half of the population eats at least one serving of fruits/vegetables each day, which is much less the five servings per day recommended by the United States Department of Agriculture [11]. The data available indicates that low fruit and vegetable intake, thus low phytonutrient intake, is correlated with excess adiposity.

The data in this study demonstrate a strong correlation between higher phytonutrient intake and improved body composition (and disease prevalence). However, this correlation remained consistent despite the type of phytonutrients consumed. These findings imply that overall diet quality seems to make the most difference, but the phytonutrients in fruits/vegetables are thought to be a key reason for those benefits.

The study of phytonutrients is difficult and expensive, which is why the PI is used in research studying health and intake of phytonutrient-rich foods [25, 28]. A major strength of the study was the ability to have a registered dietitian meet with each participant face-to-face to conduct the dietary recalls. In comparison to a voice call or participant filled record, the face-to-face recalls allowed for more follow-up questions and visual aids to more precisely determine food intake. Another strength of the study was the use of three different measures of body composition. The study used BMI, waist circumference, and body fat percentage that in combination help more accurately determine body adiposity by offsetting any limitations that each individual measure may have $[31,33]$.

The most notable limitation of this study was the method of gathering dietary data through food recalls and food frequency questionnaires. These methods are subjective interpretations that rely on the ability of each participant to recall food and quantify food intake, which leaves room for error [23]. In addition, the food recalls could not quantify for very small intakes of 
phytonutrient rich foods (such as sesame seeds on a hamburger bun) that were part of an individual's diet. Another limitation is the inability to rule out other factors that can be contributing to better body composition such as higher fiber intake and lower calorie intake which are both related to diet high in phytonutrients [40, 41]. Another factor that could have improved this study would be to increase the sample size and vary the source of the population instead of selecting all from one facility. This would not only allow for a larger data set, but potentially more diverse data as well.

There have been very few clinical intervention trials on phytonutrient intake that have been properly designed and executed due to these studies being difficult to conduct and expensive [18, 23]. However, data from correlational studies like this do suggest a role for phytonutrients in the prevention/treatment of obesity. Future studies should adopt an observational design that monitors the intakes and body composition of individuals over time to see if changes in either or both support the role of these nutrients. In addition, future studies should also observe the health conditions in participants to see if these phytonutrients have a role in prevention/treatment of diseases. In the future, new and more sufficient models are likely to be developed that would allow the design to be more consistent and accurate in the way data can be more objectively measured. Until those tools are developed, researchers should continue to use nutrition experts to recall intakes with participants in person to determine nutrition intake as accurately as possible [23, 24].

\section{CONFLICT OF INTEREST}

The authors do not have any financial or conflicts of interest.

\section{ABBREVIATIONS}

PI, phytonutrient index; BMI, body mass index; ANOVA, analysis of variance; F/V, fruits and vegetables; BIA, bio-impedance analysis; FFQ, food frequency questionnaire.

\section{ACKNOWLEDGEMENTS AND FUNDING}

We acknowledge Louisiana Tech University for study design resources and publication funding. We also acknowledge Thibodaux Regional Medical Center for access to facility for data collection.

\section{REFERENCES}

1. Ard JD, Miller G, Kahan S: Nutrition interventions for obesity. Med Clin North Am 2016, 100(6):1341-1356

2. Rolls BJ: The relationship between dietary energy density and energy intake. Physiol Behav 2009, 97(5): 609-615

3. Hill JO, Wyatt HR, Peters JC: Energy balance and obesity. Circulation 2012, 126(1): 126132.

4. Mytton OT, Nnoaham K, Eyles H, et al.: Systematic review and meta-analysis of the effect of increased vegetable and fruit consumption on body weight and energy intake. BMC Public Health 2014, 14:886-897. DOI: https://doi.org/10.1186/1471-2458-14-886

5. Swinburn BA, Caterson I, Seidell JC, James WP: Diet, nutrition and the prevention of excess weight gain and obesity. Public Health Nutr 2004, 7(1A):123-146 
6. Carnauba RA, Chaves DF, Baptistella AB, et al.: Association between high consumption of phytochemical-rich foods and anthropometric measures: a systematic review. Int J Food Sci Nutr 2017, 68(2):158-166.

7. Rodriguez-Casado A: The health potential of fruits and vegetables phytochemicals: Notable examples. Crit. Rev. Food Sci. Nutr 2016, 56(7):1097-1107. DOI: https://doi.org/10.1080/10408398.2012.755149

8. Adult Obesity facts - Overweight \& Obesity Centers [http://www.cdc.gov/ obesity/data/adult.html.]

9. Louisiana State of Obesity Data, Rates, and Trends [www.stateofobesity.org/states/la/]

10. Louisiana State Nutrition, Physical Activity, and Obesity Profile [www.cdc.gov/nccdphp/dnpao/state-local-programs/profiles/louisiana.html.]

11. Fruit and Vegetable Recommendations. USDA ERS - Food Environment Atlas. [www.ers.usda.gov/amber-waves/2016/march/fruit-and-vegetable-recommendations]

12. Liu, RH: Health Benefits of Phytochemicals in Whole Foods: From Nutritional Health: Strategies for Disease Prevention. 3rd ed. Edited by Temple NJ, Wilson T, Jacobs DR. New York, NY: Humana Press; 2012: 293-310.

13. Pizzorno, L: Highlights from the Institute for Functional Medicine's 2014 Annual Conference: Functional Perspectives on Food and Nutrition: The Ultimate Upstream Medicine. Integr Med (Encinitas) 201, 13(5): 38-50.

14. Slavin JL, Lloyd B: Health benefits of fruits and vegetables. Adv Nutr 201, 3(4): 506-516. DOI: https://doi.org/10.3945/an.112.002154.

15. Cook LT, O'Reilly GA, Goran MI, Weigensberg MJ, Spruijt-Metz D, Davis JN: Vegetable consumption is linked to decreased visceral and liver fat and improved insulin resistance in overweight Latino youth. J Acad Nutr Diet 2014, 114(11): 1776-83. DOI: https://doi.org/10.1016/j.jand.2014.01.017.

16. Stenblom EL, Weström B, Linninge C, et al.: Dietary green-plant thylakoids decrease gastric emptying and gut transit, promote changes in the gut microbial flora, but does not cause steatorrhea. Nutr Metab (Lond) 2016, 13: 67.

17. Szulinska M, Gibas-Dorna M, Miller-Kasprzak E, et al.: Spirulina maxima improves insulin sensitivity, lipid profile, and total antioxidant status in obese patients with welltreated hypertension: A randomized double-blind placebo-controlled study. Eur Rev Med Pharmacol Sci 2017, 21(10): 2473-2481.

18. Azzini E, Giacometti J, Russo GL: Antiobesity effects of anthocyanins in preclinical and clinical studies. Oxid Med Cell Longev 2017, 2740364. DOI: https://doi.org/ $10.1155 / 2017 / 2740364$

19. Meydani M, Hasan ST. Dietary polyphenols and obesity. Nutrients 2010, 2(7): 737-51.

20. Williams DJ, Edwards D, Hamernig I, et al.: Vegetables containing phytochemicals with potential anti-obesity properties: a review. Food Res Int 2013, 52(1): 323-33.

21. Bonet ML, Canas JA, Ribot J, Palou A: Carotenoids in Adipose Tissue Biology and Obesity. Subcell Biochem 2016, 79: 377-414. 
22. Whigham LD, Valentine AR, Johnson LK, Zhang Z, Atkinson RL, Tanumihardjo SA: Increased vegetable and fruit consumption during weight loss effort correlates with increased weight and fat loss. Nutr Diabetes 2012. 2(10): e48. doi:10.1038/nutd.2012.22

23. Probst YC, Guan VX, Kent KL: Dietary phytochemical intake from foods and health outcomes: a systematic review protocol and preliminary scoping. BMJ Open 2017, 7: e013337. DOI: https://doi.org/10.1136/bmjopen-2016-013337.

24. Gonzalez-Castejon M, Rodriquez-Casado A. Dietary phytochemicals and their potential effects on obesity: A review. Pharmacol. Res 2011, 64(5): 438-455. DOI: https://doi.org/10.1016/j.phrs.2011.07.004.

25. McCarty MF: Proposal for a dietary "phytochemical index". Med Hypotheses 2004, 63(5): 813-7

26. Bahadoran Z, Golzarand M, Mirmiran P, Saadati N, Azizi F: The association of dietary phytochemical index and cardiometabolic risk factors in adults: Tehran lipid and glucose study. J Hum Nutr Diet 2013, 26(Suppl 1): 145-53.

27. Mirmiran P, Bahadoran Z, Golzarand M, Shiva N, Azizi F: Association between dietary phytochemical index and 3-year changes in weight, waist circumference and body adiposity index in adults: Tehran lipid and glucose study. Nutr Metab (Lond) 2012, 9(1): 108.

28. Vincent HK, Bourguignon CM, Taylor AG: Relationship of the dietary phytochemical index to weight gain, oxidative stress and inflammation in overweight young adults. J Hum Nutr Diet 2010, 23(1): 20-9.

29. Shah NR., Braverman ER: Measuring adiposity in patients: the utility of body mass index (BMI), percent body fat, and leptin. PLoS One 2012, 7(4): e33308. DOI: https://doi.org/10.1371/journal.pone.0033308.

30. Nuttall FQ: Body mass index: obesity, BMI, and health. A Critical Review. Nutr Today 2015, 50(3): 117-128. DOI: https://doi.org/10.1097/NT.0000000000000092.

31. Borruel S, Molto JF, Alpanes M, et al.: Surrogate markers of visceral adiposity in young adults: Waist circumference and body mass index are more accurate than waist hip ratio, model of adipose distribution and visceral adiposity index. PLoS One 2014, 9(12): e114112. DOI: https://doi.org/10.1371/journal.pone.0114112.

32. van Dijk SB, Takken T, Prinsen EC, Wittink H: Different anthropometric adiposity measures and their association with cardiovascular disease risk factors: a meta-analysis. Neth Heart J 2012; 20(5): 208-18.

33. Yu Y, Wang L, Liu H, et al.: Body mass index and waist circumference rather than body adiposity index are better surrogates for body adiposity in a Chinese population. Nutr Clin Pract 2015, 30(2): 274-282. DOI: https://doi.org/10.1177/0884533614564468.

34. Kyle U.G., Piccoli A., Pichard C: Body composition measurements: Interpretation finally made easy for clinical use. Curr Opin Clin Nutr Meta. Car. 2003, 6(4): 387-393. doi: 10.1097/01.mco.0000078988.18774.3d.

35. Miyatani M, Yang P, Thomas S, Craven BC, Oh P: Bioelectrical impedance and dualenergy $\mathrm{x}$-ray absorptiometry assessments of changes in body composition following 
exercise in patients with type 2 diabetes mellitus. J Obes 2012, 953060. DOI: https://doi.org/10.1155/2012/953060.

36. Stahn A, Terblanche E, Gunga HC: Use of Bioelectrical Impedance: General Principles and Overview: From Handbook of Anthropometry. Edited by Preedy V. New York, NY: Springer; 2012. DOI: https://doi.org/10.1007/978-1-4419-1788-1_3

37. Usual Dietary Intakes - NHANES Food [epi.grants.cancer.gov/diet/usualintakes/ffq.html.]

38. Cade J, Thompson R, Burley V, Warm D: Development, validation and utilisation of foodfrequency questionnaires-A review. Public Health Nutr 2002, 5(4):567-587. DOI: https://doi.org/10.1079/PHN2001318.

39. World Health Organization (WHO): Waist circumference and waist-hip ratio: Report of a WHO expert consultation. WHO STEPwise Approach to Surveillance (STEPS) 2008.

40. Ledoux TA, Hingle MD, Baranowski T: Relationship of fruit and vegetable intake with adiposity: a systematic review. Obes Rev 2011, 12(5): e143-e150. DOI: https://doi.org/10.1111/j.1467-789X.2010.00786.x

41. Schwingshackl L, Hoffmann G, Kalle-Uhlmann T, Arregui M, Buijsse B, Boeing H: Fruit and vegetable consumption and changes in anthropometric variables in adult populations: A systematic review and meta-analysis of prospective cohort studies. PLoS One 2015, 10(10): 1-19.

42. Aune D, Giovannucci E, Boffetta P, et al.: Fruit and vegetable intake and the risk of cardiovascular disease, total cancer and all-cause mortality-A systematic review and dose-response meta-analysis of prospective studies. International Journal of Epidemiology 2017, 46(3): 1029-1056.

43. The role of fruit and vegetable consumption in human health and disease prevention From Diabetes Mellitus- Insights and Perspectives- IntechOpen [http://www.intechopen .com/books/diabetesmellitus-insights-and-perspectives/the-role-of-fruit-and-vegetableconsumption-in-humanhealth- and-disease-prevention]

44. Pem D, Jeewon R: Fruit and vegetable intake: Benefits and progress of nutrition education interventions- Narrative review article. Iran. J Public Health 2015, 44(10): 1309-1321.

45. Dehghan M, Akhtar-Danesh N, Merchant AT: Factors associated with fruit and vegetable consumption among adults. J Hum Nutr Diet 2011, 24(2): 128-134. DOI: https://doi.org/10.1111/j.1365-277X.2010.01142.x.

46. Halkjaer J, Tjønneland A, Overvad K, and Sørensen TA: Dietary predictors of 5-year changes in waist circumference. J Am Diet Assoc 2009, 109(8): 1356-1366.

47. Bertoia ML, Mukamal KJ, Cahill LE, et al.: Changes in intake of fruits and vegetables and weight change in United States men and women followed for up to 24 years: Analysis from three prospective cohort studies. PLoS Med 2015, 12(9): e1001878. DOI: https://doi.org/10.1371/journal.pmed.1001878.

48. Zhao J: Nutraceuticals, nutritional therapy, phytonutrients, and phytotherapy for improvement of human health: A perspective on plant biotechnology application. Recent Patents in Biotechnology 2007, 1(1): 75-97. 